Table III.-Shows the causes of the deaths which took place among the horses during a period of eight years.

Fatal Diseases and Accidents.

Died.

Diseases of the brain (staggers)...... 4

Lnck-jaw (trismus) ............. 2

Disease of the lungs............. 23

Hydrothorax (water in the chest)..., 1

Disease of the abdominal viscera .... 3

Shot on account of

Farcy ................... 3

Glanders ................ 6

Fracture of a leg............ 6

a coffin bone......... 1

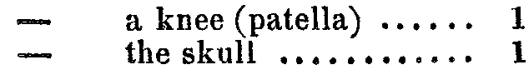

- the pelvis ............ 1

- the haunch bone...... 2

Injury of the spine by a fall ......

Injury by a cart $\ldots \ldots \ldots \ldots \ldots \ldots 1$

Total Deaths......58

From this table it is obvious that affection of the lungs is by far the most fatal disease among caralry horses. It shows that twofifths of the mortality has a pulmonary origin.

TABLE IV.-Shows the Number of Horses

Cast during elght years; together with the Disabilities which rendered them unfit for Cavalry Duty.

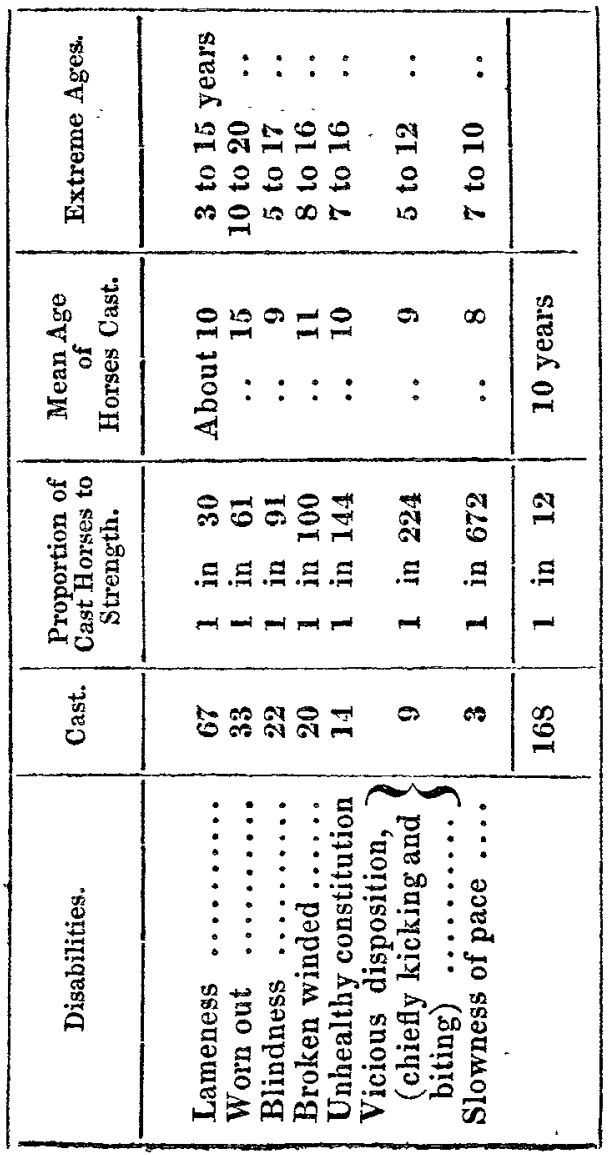

With respect to the mean age of the horses cast, as stated in the above table, I may observe that it is 10 years; and that the mean age of the horses in 1836 was 9 , and in $1837,9 \frac{1}{3}$ years.

Cast horses, although unfit for cavalry duty, may be useful for many other purposes. They are commonly sold by public auction, and bring, on an average, about $8 l$. or $9 l$. each.

\section{ENORMOUS TUMOUR OF THE UPPER JAW}

SUCCESSFULLY REMOVED BY OPERATION.

$B y$ R. O'Shaughnessy, Esq., M. $R$. C. $S$. Demonstrator of Anatomy, Medical College, Calcutta, and Superintendent of the Gurran. hatta Dispensary.

As tumours of the jaws have lately at. tracted much of the attention of some of the most distinguished surgeons in Europe, and as $I$ have been given to understand that they are anything but uncommon in this country (though I cannot discover that tumours of any considerable size have, before the present instance, been removed from that region by any surgeon in India), I trust that the following case may be deemed wor. thy the notice of the Society, and perhaps, a place in their valuable $J$ ourual.

Woodey Morrol, æt. 21, a Hindu of the farmer caste, tall, thin and slightly emaci. ated, native of a village called Pangey, in the district of Kasba, which is a day's march from Jessore, and about three days' journey from Calcutta, presented himself at the Gurranhatta Dispensary on the 6th of $\mathrm{N}_{0}$ vember, 1837, under the following circumstances.

He says that about a year ago a swelling formed in his left cheek, immediately above the second grinder, about the size of a sparrow's egg, causing much pain and inconvenience; that it gradually increased in size, and about four months from its rom= mencement, it had attained the bulk of a large orange, when he sought relief from a native doctor, who told bim it was an abscess, which he promised to cure as soon as he thought it soft enough to puncture, and accordingly, in three or four days, though the hardness of the tumour did not at all diminish, he commenced his treatment by thrusting a needle into it, but no matter flowed from the wound thus formed; the doctor then set to work to bring it to a head, and for a few days more, frequently rubbed it with some mysterious compond which he appeared to prepare with great skill and care; but this disappointing his expecta. tions also, he gave it up, and absconded. The puncture made into the swelling with the needle produced no ulcer or fungus, though the operation caused great pain and suffering to the patient.

From the time the doctor ceased his treat. 
ment the tumour has gone on increasing; it gradually protruded into the mouth, and six months after its first appearance it commenced bleeding copiously once or twice a month, and he says the bleeding was more abundant and more certain to return at the full of the moon than at any other time during the month. This periodical discharge of blood did not produce any salutary alteration, or effect any check on the advancement of this frightful disease; it still continued to increase in pain and bulk till after filling the mouth so as nearly to produce suffocation, it at last (about six weeks ago) protruded from that cavity through the lips, and went on rapidly increasing up to the present day.

He positively says that he never received an injury of any kind in that cheek or jaw, and that he never had a tooth drawn or an un. sound one. On his admission into the Dispensary, the tumour presented the following appearance.

An enormous growth completely occu. pied the left side of the face, rising to a level with the floor of the orbit and extending a long way below the inferior maxilla but unattached to it, occupying the whole of the anterior and left side of the mouth and protruding between the lips, pressing down the lower jaw, so as almost to make the chin touch the throat, and flattening the nose so as to leave no trace of the natural prominence of that organ. Still there was difficulty of swallowing, and the patient seemed to breath without inconvenience through the right nares. That portion of the tumour which protruded through the mouth, was of a bright red color and covered with mucous membrane, having at its upper part the canine and the two incisors of its own side, with the central incisor of the opposite maxilla, sticking out of it. The dimensions of this mass were as follows: from the part near the ear to the most prominent point which protruded from the mouth, exactly 12 inches, and from that part which bulged below the inferior maxilla to the edge of the orbit, about 10 inches. It looked, as near as may be, equal in size to the patient's head. The skin over the tumour was perfectly sound and not adhering to it, and many of the muscles of that cheek still retained their healthy actions; there was not the slightest trace of ulceration on any part of the tumour, and the principal source of pain to the patient appeared to be from distention and pressure on the surrounding parts.

He always hung'a cloth upon the tumour, the end of $\mathrm{x}$ hich he kept constantly applied to his mouth for the purpose of collecting the saliva which was secreted in great abundance, and also to concentrate the sound of the voice when speaking.

Taking everything into consideration, the youth of the patient, and his general good health, and also the benign character of the tumour, and its freedom from any attachment to the lower jaw, I felt not only warranted but in duty bound to offer to this

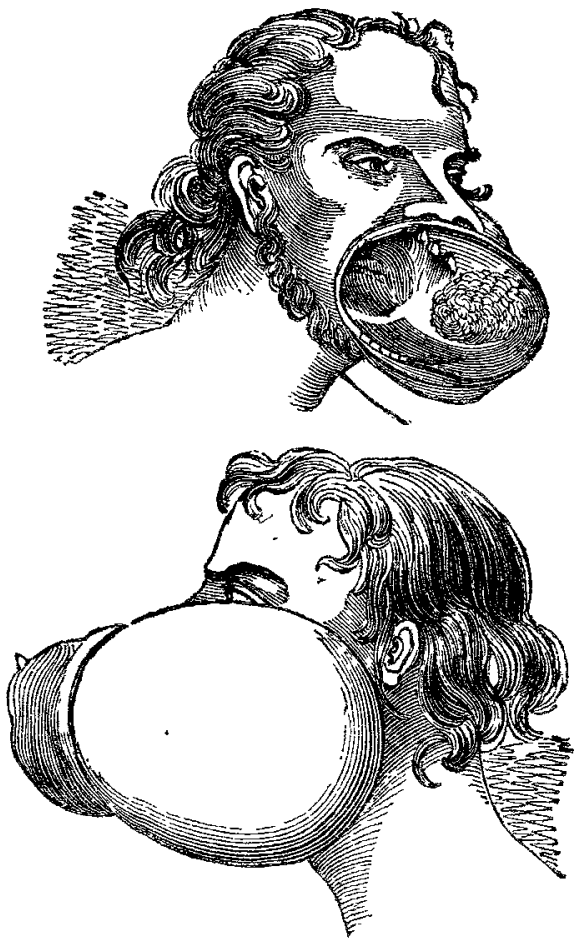

poor sufferer the only chance now left for him of escaping a lingering and frightful death, which of course was only to be hoped for by his submitting to an operation, and he not only willingly, but joyfully acceded to the proposal.

I must here mention that what gave me greatest confidence-in fact, what made me feel almost certain of success was the perusal of a paper by Mr. Liston on "tumours of the month and jaws," in which he states several cases of enormous tumours of the upper jaw operated on by himself, and so successfully, that I did not doubt for a moment as to the issue of this case, as I considered my patient a much more favourable one to operate upon than any of those whom Mr. Liston mentions, though at the same time, the tumour was much larger than any removed by that justly celebrated surgeon, or indeed by any other surgeon in Europe, so far as I can ascertain.

On the 9th, three days after the patient was admitted into the Dispensary, I had him taken to the theatre of the Medical College, where, for the advantage of the pupils, and also as having there better light and room than in any apartment in my dispensary, I thought it best to operate.

The patient being seated in a stout arm chair, and his head supported by Dr. Corbyn, who kindly offered to undertake that charge, while a second assistant, Dr. Goodeve, stood at his left side prepared to make 
pressure on the carotid artery, if at any time during the operation the bleeding called for such interference, I commenced the operation by making a cut through the skin over the upper part of the tumour, commencing at the posterior edge of the left malar bone and terminating in the upper lip, which I divided about an inch from the corresponding ala of the nose. I then cut fiom its bony attachments the cartilage of the nose, turned up the left ala and continued the dissection as far upwards as the edge of the orbit, and back to the zygomatic process of the malar bone, which I laid bare, and with a Liston's bone-nippers divided. I next carefully raised the periosteum of the foor and external side of the orbit with the handle of the knife, and again took the bone-nippers and cut through the malar bone into the spheno-maxillary fissure. I then cut through the orbital process of the superior maxilla with a strong knife, dividing the superior maxillary nerve at the same time; the nasal process of the superior maxilla was next cut, and then, after drawing the second incisor of the opposite side (for the extent of the disease required it), I cut through the alveolar process, and hard palate, as far back as the palatal process of the palate bone, with the bone-nippers; and now all the strong at. tachments of the tumour being completely severed, I had no difficulty in removing that mass, carefully separating with the knife the palatal process of the superior maxilla from the palatal plate of the palate bone, so as to preserve the soft palate from injury. The whole of the superior maxillary bone of the left side, part of the alveolar processes and palate plate of the superior maxilla of the opposite side, and also the malar bone of the left side, were involved in the disease.

The tumour weighed four pounds, it was nearly globular in form, having at its inferior surface a deep groove into which the lower jaw sunk, and the teeth before mentioned projecting from its anterior and upper part. In making a section right through its axis it was found to be of a dense fibro. cartilaginous structure surrounded for threefourths of its entire extent by a pellicle of bone about the thickness of fine parchment, and where the bone was deficient by condensed mucous membrane.

During the whole time of the operation, which took near 10 minutes to complete, there was not the slightest need for interfer:ence with the carotid artery, or even to place a finger on a spouting vessel; and $I$ should suppose, in all, the patient conld not have lost more than from 8 to $9 \mathrm{oz}$. of blood; no ligatures were required, and a few minutes after the tumour was removed all bleeding ceased. Not a particle of the disease could have been left behind as the tumour came out whole and unbroken, ex cept a small piece which pressed so high up against the floor of the orbit, that I was obliged to cut off when removing the great mass; but this came out immediately after without even having to use the knife for its removal.

I prit a few dossils of lint into, and brought the edges of the wound together with five points of interrupted and three of the twisted sutures. As soon as this part of the opetion was finished the patient appeared to be slightly convulsed, and fainted ; he was then laid on his back on the floor but after a few seconds revived again, when he sat up him. self, and insisted on being allowed to swal. low two or three tumblers of cold water ; he was then placed in bed and a dossil of wet lint laid over the wound. I had given him 80 drops laudanum before the operation, but in about two hours after it, as he complained loudiy of pain, 1 gave him $1 \mathrm{gr}$. mur. morphiæ, which set him to sleep after a short time.

9 P. м. Complaining of great pain, but in every respect is getting on satisfactorily; no oozing of blood from the wound, pulse 120, small, slight heat of skin, the wet lint to be kept constantly to the wound.

10. 6 A. M. Passed a good night, slept several hour's and did not complain so much when he awoke as before falling asleep, pulse 100, still very small; heat of skin slightly increased, wound looking well, slight swelling and inflammation of the cheek.

12 o'clock, twenty-four hours after the operation. Complaining of great pain, but the lower part of wound united by the airst intention; to take immediately $1 \mathrm{gr}$. mur. morphia.

9 P. M. Slept several hours after taking the morphia, and when he awoke folt so much relieved as to insist on being permitted to smolie the huqqa, which the attendants say he managed very well with the sound side of his month; had two healthy stools in the course of the day; heat of skin not so much as it was in the morning; to get $11 \mathrm{gr}$. mur. morphia at 11 o'clock to-night.

11. 6 A. м. Passed a good night, slept soundly for several hours; wound looking well and nearly all united, swelling of face increased, heat of skin higher than yesterday, and he complained of pains all over lis body: misturæe sennæ 1 oz. to be taken immediately.

8. P. Mr. The medicine operated on his bowels three or four times, and he has since been much relieved; has now no fever and only complains of the soreness of his month; ate some sago in the course of the afternoon and fornd no difficulty in swallowing.

12. Three days after the operation, wound of face quite healed except in one small point below the external angle of the eye; removed all the pins and sutures, and dressed the part with strips of isinglass plaster, The upper extrenity of wounl looks 
much infamed and as if likely to slough. | may safely conclude, that at least there is There is much oedema and swelling of the no probability of a return of the disease in lip, both above and below the part divided; flow of saliva from the mouth most profuse, so much so as to oblige him to keep his head constantly over a vessel to receiveit: ordered some infusion of oak bark as a wash for mouth. I should have mentioned that the mouth remained as wide apart after the operation as before the tunour that distended it was removed; he appeared to have lost the power over the muscles that raise the lower jaw, but it is gradually closing, and I dare say, in a day or two more be will be able to shut it completely.

13. Slept well last night, no ferer, and now makes but few complaints: the flowing from the mouth still very profuse.

14. No change, except that he can now shut his mouth.

15. The plaster got loose, and about an inch and a half of upper part of wound, which was united, has separated; a small slough has also come away from its upper corner, but the wound inside is filling up rapidly with healthy granulations. Patient's general health improving, pulse strong and regular, and he eats with a good appetite; brought the edges of the wound together again with some strips of common adhesive plaster, which I now think preferable for such wounds ; odema of lower part of face diminishing, and the flowing from the mouth not so profuse; skin contracting very much, and thickening.

20. Has been getting on very well, odema of lower lip much reduced, and the mouth is now nearly as small as it ever could have been. He has perfect action over the obicularis oris at the wounded as well as the sound side of the mouth; the upper part of wound contracting slowly but not y't united; he is looking stouter than he was before the operation.

I may observe, before concluding this communication, that I consider this case as characterised by every mark which distinguishes the benign from the malignant form of tumour of the upper jaw. The skin was sound and unadhering to the tumour, the tumour itself was firm, and not painfal to the touch, the patient's constitution was unimpaired, and the expression of his countenance was healthy; while, on the other hand, all the signs of malignancy were absent, there was no trace of unhealthy ulceration, or of the soft and bleeding fungi making their way through the integuments, no fetid discharge tinged with blood; nor were there any purple tubercles adhering to the subjacent tumour to be seen upon the skin, as occurs in cases of an unhealthy character; so I think, if I am to judge by the experience of those surgeons who have given much attention to the diseases of the jaws, and have published their opinions and the result of their observationg, that $I$

There is one point worthy of remark in this case, that is, the rapidity of its growth, for we are told, that the progress of the benign tumours of the jaw is slow as well as painless; that, in fact, it is not till after several years existence they are found of any very considerable size; but I believe this statement only holds good as referring to the disease in Europeaus, as I have been informed by several surgeons who have long resided in this country, that the rapid progress of such tumours in the natives of Bengal and the Upper Provinces is truly astonisbing.

On the 14th of December, exactly five weeks after the operation, this man was discharged, perfectly cured, and a likeness of him taken on the day before he left the hospital.-Calculta Quarteriy Journal, Jan. 1838.

\section{IMPERFORATION OF THE ANUS.}

\section{To the Editor of The Lancet.}

SIR:-I use the license of an old correspondent, to forward to you a statement of what I consider to be an interesting case of impervions rectum; the patient a fine healthy boy.

No stools having passed for two days, an examination was made. The anus was perfectly developed, the sphincter acting strongly when excited; and the rectum naturally pervious to the extent of three fourths of an inch, at which point all track of the channel was perfectly obliterated; it felt as if the lower portion of the bowel had grown to the sacrum, on which part the distended bladder could be distinctly felt. A few days having elapsed withont any prospect of change, I requested the assistance of Mr. Salmon, who concurred in the propriety of waiting until a larger col lection of freces should be accumulated, or untoward symptoms demand an operation.

The child sucked heartily, and slept soundly, exhibiting no signs of distress, and nature appeared to have set up a process for the amelioration of the mischief by copious perspiration, extraordinary secretion of urine, and accumulation of fat; there was likewise occasional vomiting.

On the eleventh day, the abdomen being somewhat tumefied, the operation was resolved on ; at the last examination, no difference was perceived in the state of the parts from that of the first day; the obliteration was thought to extend so high, that the hoped for bagging of the faces collected above the obliterated portion of the intestine, could not be ccrtainly distinguished.

Mr. Salmon operated by introducing a trocat of the full gige of a swan-guill, which 